\title{
New tests for high-energy factorization in hard hadron collisions
}

\author{
N.P. Zotov ${ }^{* \dagger}$ \\ Skobeltsyn Institute of Nuclear Physics, Lomonosov Moscow State University \\ E-mail: zotovatheory.sinp.msu.ru
}

\section{S.P. Baranov}

Lebedev Insitute of Physics, Russian Academy of Science

E-mail: baranovasci.lebedev.ru

\section{A.V. Lipatov}

Skobeltsyn Institute of Nuclear Physics, Lomonosov Moscow State University

E-mail: lipatovetheory.sinp.msu.ru

\begin{abstract}
We study the production of Drell-Yan lepton pairs at the Tevatron in the framework of the $k_{T^{-}}$ factorization approach. We use the unintegrated, or transverse momentum dependent (TMD) quark density in a proton, obtained from the TMD valence and sea quark distributions. The latter is calculated as a convolution of the CCFM-envolved gluon distribution and TMD gluon-to-quark splitting function (occurs at the last evolution step). Our numerical predictions are compared with the data taken by the D0 and CDF collaborations at the Tevatron energies
\end{abstract}

The XXI International Workshop High Energy Physics and Quantum Field Theory,

June 23 - June 30, 2013

Saint Petersburg Area, Russia

\footnotetext{
* Speaker.

$\dagger$ The authors were supported by RF FASI grant NS-3920.2012.2, RFBR grants 12-02-31030 and 13-02-01060, the grant of the Ministry of Science and Education (8412) and also by DESY Directorate in the framework of Moscow DESY project on Monte-Carlo implementation for HERA - LHC.
} 


\section{Introduction}

The production of Drell-Yan lepton pairs at energy of modern colliders is a subject of intense studies from both experimental and theoretical points of view [1]. First of all, Drell-Yan pair production is a unique process which offers a high sensitivity to the quark distribution in a proton. Second, with help of investigation of Drell-Yan pair production in the LHC forward region it is possible to study QCD dynamics at very small $x$ (up to $x \sim 10^{6}$ ), where non-linear gluon interactions are expected to become more and more important, resulting eventially in the slowdown of the gluon density growth (known as "saturation effects") [2, 3]. Study of Drell-Yan process at TMD level is based on the non-collinear (TMD) factorization theorem for this process, which has been proven [ $₫$ ] for $p_{T}<<M$ ( $\mathrm{M}$ is lepton pair mass) and the definition for TMDs determined by requirement of factorization, maximal universality and internal consistency have been done by Collins [5]. Below we assume it in a wide range of $p_{T}$ for phenomenological purposes.

First application of $k_{T}$-factorization approach to lepton pair production has been performed in [6], where authors have considered only diagrams with virtual photon exchange and concentrated mostly on the rather low energies covered by the RHIC and UA1 experiments. Our previous consideration [7] has been based on the $O(\alpha)$ and $O\left(\alpha \alpha_{s}\right)$ matrix elements, where we took into account off-shell gluons and both $\gamma$ and $\mathrm{Z}$ boson exchanges ${ }^{1}$. We have used the KimberMartin-Ryskin unintegrated parton distrubutions. However for a Monte Carlo implementation of $k_{T}$-factorization, which is realized by the Monte Carlo event generator CASCADE [9], the Ciafaloni-Catani-Fiorani-Marchesini (CCFM) evolution equation [10] have been solved and the CCFM unintegrated gluon distributions have been obtained. Here we demonstrate the impact of some of the details of the behavior of these functions on the description of experimental data of Drell-Yan lepton pair production.

\section{Theoretical framework}

According to the $k_{T}$-factorization theorem, in order to calculate the cross section of Drell-Yan pair production one should convolute off-shell partonic cross sections with the relevant unintegrated quark distributions in a proton:

$$
\sigma=\sum_{i, j=q, g} \int \hat{\sigma}_{i j}\left(x_{1}, x_{2}, \mathbf{k}_{1 T}^{2}, \mathbf{k}_{2 T}^{2}\right) f_{i}\left(x_{1}, \mathbf{k}_{1 T}^{2}, \mu^{2}\right) f_{j}\left(x_{2}, \mathbf{k}_{2 T}^{2}, \mu^{2}\right) d x_{1} d x_{2} d \mathbf{k}_{1 T}^{2} d \mathbf{k}_{2 T}^{2},
$$

where $\hat{\sigma}_{i j}\left(x_{1}, x_{2}, \mathbf{k}_{1 T}^{2}, \mathbf{k}_{2 T}^{2}\right)$ is the relevant partonic cross section. The initial off-shell partons have fractions $x_{1}$ and $x_{2}$ of initial protons longitudinal momenta and non-zero transverse momenta $\mathbf{k}_{1 T}$ and $\mathbf{k}_{2 T}$.

We take into account here Drell-Yan annihilation subprocesses in the leading order only: $q^{*}+q \rightarrow \gamma^{*} / Z \rightarrow e^{-}+e^{+}$. Evaluation of the off-shell matrix elements is rather straightforward (sm. [7]). We used standard Feynman rules. In order to avoid artificial singularities in numerical calculations, for Z-boson propagator we took Breit-Wigner form. Since the incoming partons are off-shell and obtain mass, proportional to their transeverse momentum squared, their polarization

\footnotetext{
${ }^{1}$ In our paper we took into account on-shell quarks in the subprocesses $q \bar{q} \rightarrow \gamma^{*} / Z \rightarrow l^{+} l^{-}$and $q g^{*} \rightarrow \gamma^{*} / Z \rightarrow l^{+} l^{-}$ with contrast claimed the paper [8].
} 
tensor should differ from the one, taken for on-shell particles. In paper [11] the spin density matrix for the off-shell quark with the momentum $k=x P+k_{T}$ in massless limit has been taken in so called in "small $x$ approximation":

$$
\sum_{s} u^{s}(k) \bar{u}^{s}(k)=x \hat{P}
$$

where $P$ is the momentum of the incoming proton (or antiproton). Since the expression was obtained in the massless approximation, we neglected the quark masses.

Here, by analogy with effective quark-to-photon vertex for reggeized quarks [12]:

$$
\Gamma_{\gamma}^{\mu}\left(k_{1}, k_{2}\right)=\gamma^{\mu}-\hat{k}_{1} \frac{P_{1}^{\mu}}{P_{1} \cdot k_{1}}-\hat{k}_{2} \frac{P_{2}^{\mu}}{P_{2} \cdot k_{2}},
$$

where $k_{1}, k_{2}, P_{1}$ and $P_{2}$ are the initial quark, antiquark and proton 4-momenta, we constructed effective reggeized quark-to- $\mathrm{Z}$ boson vertex:

$$
\Gamma_{Z}^{\mu}\left(k_{1}, k_{2}\right)=\Gamma_{\gamma}^{\mu}\left(k_{1}, k_{2}\right)\left(g_{V}-g_{A} \gamma^{5}\right),
$$

where $g_{V}$ and $g_{A}$ are standard vector and axial quark to $\mathrm{Z}$ boson coupling constants. In this case Ward identity is satisfied:

$$
\Gamma_{Z}^{\mu}\left(k_{1}, k_{2}\right)\left(k_{1}+k_{2}\right)_{\mu}=\Gamma_{\gamma}^{\mu}\left(k_{1}, k_{2}\right)\left(k_{1}+k_{2}\right)_{\mu}=0,
$$

and gauge invariance is achieved in our calculations.

It is known that the CCFM equation describes only the distinct evolution of TMD gluon and valence quarks, while non-diagonal transitions between quarks and gluons are absent. The TMD gluon and valence quark distributions $f_{g}\left(x, \mathbf{k}_{T}^{2}, \mu^{2}\right)$ and $f_{q}^{(v)}\left(x, \mathbf{q}_{T}^{2}, \mu^{2}\right)$ have been obtained [13] from the numerical solutions of the CCFM equation. In the approximation where the sea quarks occur at the last gluon-to-quark splitting, the TMD sea quark density at the next-to-leading logarithmic accuracy $\alpha_{s}\left(\alpha_{s} \ln x\right)^{n}$ can be written[14] as follows:

$$
f_{q}^{(s)}\left(x, \mathbf{q}_{T}^{2}, \bar{\mu}^{2}\right)=\int_{x}^{1} \frac{d z}{z} \int d \mathbf{k}_{T}^{2} \frac{1}{\triangle^{2}} \frac{\alpha_{s}}{2 \pi} P_{q g}\left(z, \mathbf{k}_{T}^{2}, \triangle^{2}\right) f_{g}\left(x / z, \mathbf{k}_{T}^{2}, \bar{\mu}^{2}\right),
$$

where $z$ is the fraction of gluon light cone momentum which is carried out by the quark, and $\triangle=\mathbf{q}_{T}-z \mathbf{k}_{T}$. The sea quark evolution is governed by the off-shell TMD gluon-to-quark splitting function $P_{q g}\left(z, \mathbf{k}_{T}^{2}, \triangle^{2}\right)$ which reads [15]

$$
P_{q g}\left(z, \mathbf{k}_{T}^{2}, \triangle^{2}\right)=T_{R}\left(\frac{\triangle^{2}}{\triangle^{2}+z(1-z) \mathbf{k}_{T}^{2}}\right)^{2}\left[(1-z)^{2}+z^{2}+4 z^{2}(1-z)^{2} \frac{\mathbf{k}_{T}^{2}}{\triangle^{2}}\right]
$$

where $T_{R}=1 / 2$. The TMD gluon-to-quark splitting function is obtained by generalizing to finite transverse momenta, in the high-energy region, the two-particle irreducible kernels expansion [15]. Although evaluated off-shell, this splitting function is universal [15, 16]. It takes into account the small- $x$ enhanced transverse momentum dependence up to all orders in the strong coupling, and reduces to the collinear splitting function at lowest order for $\mathbf{k}_{T}^{2} \rightarrow 0$. The scale $\bar{\mu}^{2}$ is defined [14] from the angular ordering condition which is natural from the point of view of the CCFM evolution: $\bar{\mu}^{2}=\triangle^{2} /(1-z)^{2}+\mathbf{k}_{T}^{2} /(1-z)$. 

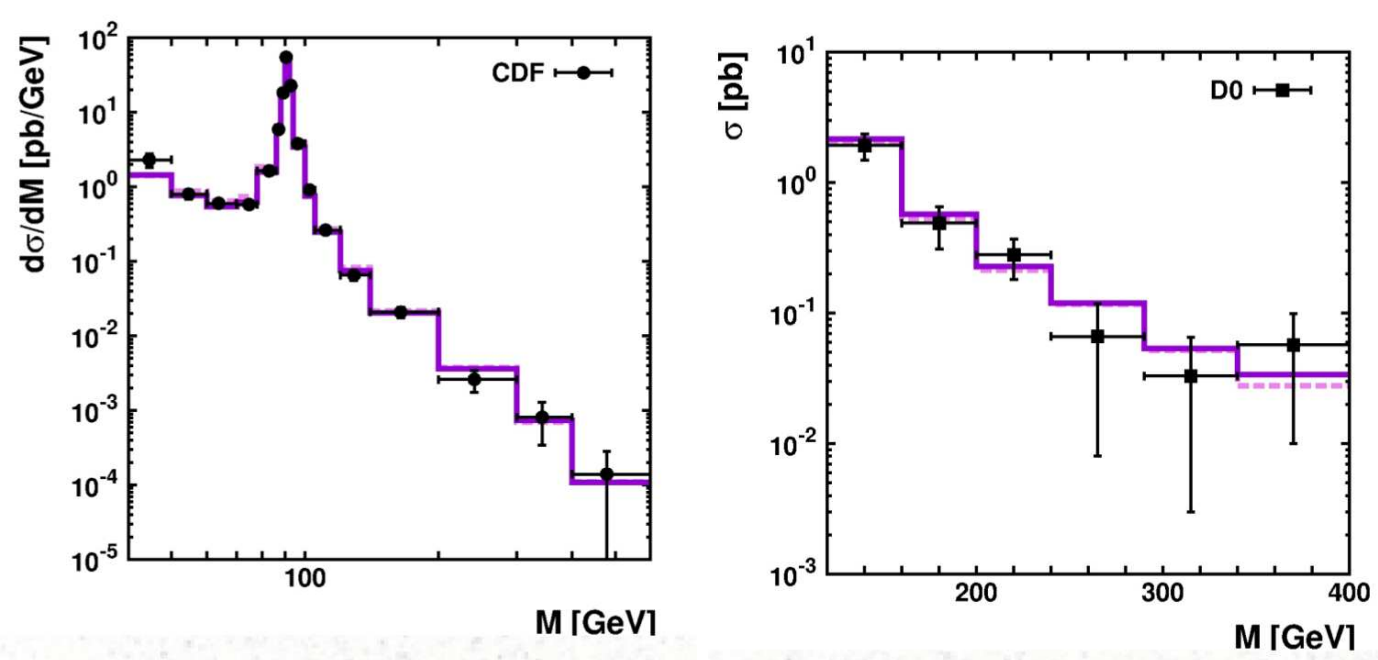

Figure 1: The differential cross sections of the lepton pair production in $p \bar{p}$ collisions as a function of $M_{e+e}$ calculated at $\sqrt{s}=1.8 \mathrm{TeV}$. The solid histograms correspond to the CCFM predictions at the default scale $\mu=M_{e+e}$, whereas the dashed histograms correspond to the results, obtained in the small-x approximation. The experimental data are from CDF [17] (left panel) and from Dり [18] (right panel).

To take into account the non-logarithmic loop corrections we use the approximation proposed in [19]. It was demonstrated that the main part of the non-logarithmic loop corrections to the quark-antiquark annihilation cross section can be absorbed in the effective $K$-factor: $K=$ $\exp \left(C_{F} \pi^{2} \alpha\left(\mu^{2}\right) / 2 \pi\right)$, where the color factor $C_{F}=4 / 3$. A particular choice $\mu^{2}=p_{T}^{4 / 3} M_{l l}^{2 / 3}$ has been proposed [19, 20] to eliminate sub-leading logarithmic terms. We chose this scale to evaluate the strong coupling constant in the expression for $K$-factor.

\section{Numerical results}

In order to calculate total and differential cross sections for dielectron production a C++ program has been written. The multidimensional integration in (2.1) has been performed by means of the Monte Carlo technique, using the routine VEGAS [21].

The renormalization and factorizaton scales were taken as $\mu_{R}=\mu_{F}=\xi M_{e^{+} e^{-}}$, where $M_{e^{+} e^{-}}$ is the invariant mass of the electron-positron pair, and the parameter $\xi$ was varied between $1 / 2$ and 2 about the default value $\xi=1$ in order to estimate the scale uncertainties of our calculations. As it was mentioned above, we neglected quarks masses. We used standard LO formula for the strong coupling constant $\alpha_{S}\left(\mu^{2}\right)$ with $n_{f}=4$ massless quark flavours and $\Lambda_{Q C D}=200 \mathrm{MeV}$, such that $\alpha_{S}\left(M_{Z}^{2}\right)=0.1232$.

In Fig. 1 (left panel) the differential cross section for the dielectron production at Tevatron at center-of-mass energy $\sqrt{s}=1.8$ in the $Z$-peak region $\left(11<M_{e^{+} e^{-}}<150 \mathrm{GeV}\right)$ is shown. The rapidity of the produced electron should have been $|y|<1$. The data was taken by CDF collaboration [17]. Then in Fig. 1 (right panel) we present the results for the simulation for D $\emptyset$ data, taken at $\sqrt{s}=1.8 \mathrm{TeV}$ in the region $120<M_{e^{+} e^{-}}<1000 \mathrm{GeV}$ [18]. The produced electron had the rapidity $|y|<1.1$ or $1.5<|y|<2.5$. The solid histograms correspond to the CCFM predictions 

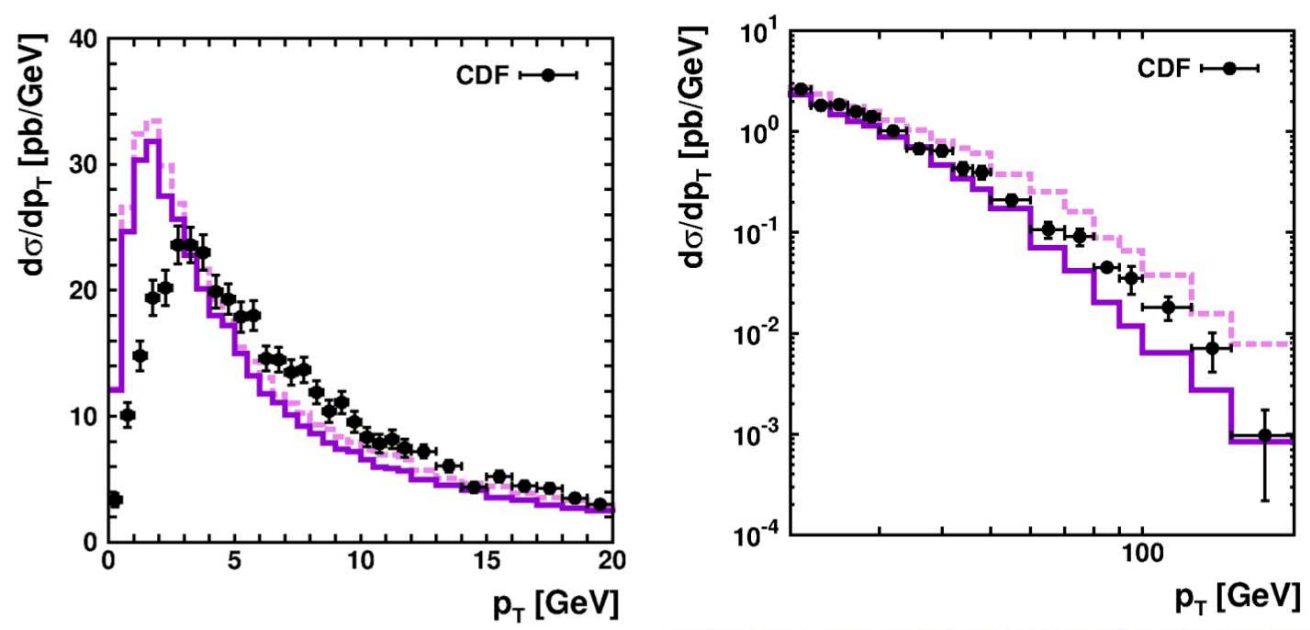

Figure 2: The differential cross sections of the lepton pair production in $p \bar{p}$ collisions as a function of $p_{T}$ calculated at $\sqrt{s}=1.8 \mathrm{TeV}$. Notation histograms as in Fig. 1. The experimental data are from CDF [22].

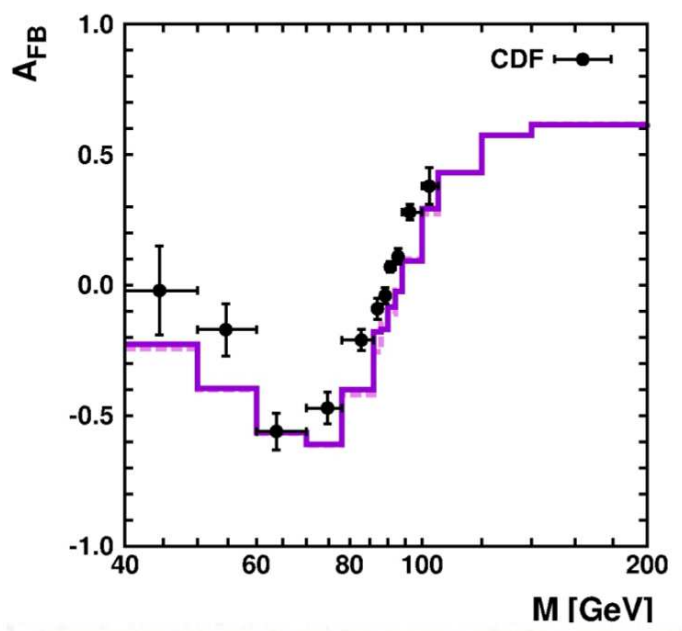

Figure 3: The assymetry of lepton pair production in $p \bar{p}$ collisions as a function of the $M_{e+e}$ calculated at $\sqrt{s}=1.8$ Tev. Notation histograms as in Fig. 1. The experimental data are from CDF [17].

with effective vertex (2.4) and splitting function (2.7) and the dashed histograms correspond to ones in small-x approximation. One can see a good description of the data in the whole range of the invariant masses.

Another result we consider is the data of CDF for $d \sigma / d p_{T}$ as a function of the transverse momentum of the Drell-Yan pair $p_{T}$ [22]. The measurements were taken at $\sqrt{s}=1.8 \mathrm{TeV}$, in $Z$ boson region $\left(66<M_{e^{+}} e^{-}<116 \mathrm{GeV}\right)$, and the produced electron had the rapidity $|y|<4.2$. The simulated results are shown on Fig. 2. For convenience, we present the results in the whole range $0<p_{T}<200 \mathrm{GeV}$ and the zoomed region $0<p_{T}<50 \mathrm{GeV}$. One can see that the description of data becomes worse as we move closer to the zero $p_{T}$, and there are the difference between the solid and dashed histograms at large values $p_{T}$. 
We finish the description of Tevatron results with the measurements by CDF of the forwardbackward assymetry of lepton pair production $A_{F B}$, taken at $\sqrt{s}=1.8 \mathrm{TeV}, 40<M_{e+e-}<200$ $\mathrm{GeV}$, and in the Drell-Yan pair rapidity region $|y|<2.9$ [17]. We present our results in Fig. 3 .

\section{Conclusion}

We have considered important process of Drell-Yan lepton pair production in the framework of the $k_{T}$-factorization QCD approach. Our consideration was based on the gauge-invariant amplitude of quark annihilation subprocess (involving Z-boson exchange) and CCFM-evolved TMD PDFs in the proton. We have used the TMD sea quark distribution and investigated the dependence of our predictions on the gluon-to-quark splitting function. We have obtained a reasonable agreement of our numerical predictions and the Tevatron data taken by the D $\emptyset$ and CDF collaborations. Furher investigations of Drell-Yan process at LHC energies are in progress.

\section{References}

[1] Z.Ajaltouni et al., arXiv:0903.3861 [hep-ph], Proceedings of the Workshop HERA and the LHC, CERN/DESY 2008.

[2] L.V. Gribov, E.M. Levin, and M.G. Ryskin, Phys. Rep. 100, 1 (1983).

[3] A.H. Mueller and J. Qiu, Nucl. Phys. B 268, 427 (1986);

K. Golec-Biernat and M. Wusthoff, Phys. Rev D 59, 014017 (1999); D 60, 114023 (1999).

[4] X.-D. Ji, J.-P. Ma and F. Yuan, Phys. Lett.B 597, 299 (2004).

[5] J.C. Collins Foundations of Perturbative QCD, Cambridge University Press, Cambridge, 2011.

[6] A. Szczurek and G. Slipek, Phys. Rev. D 78, 114007 (2008).

[7] A.V. Lipatov, M.A. Malyshev and N.P. Zotov, JHEP 1112, 117 (2011).

[8] M.A. Nefedov, N.N. Nikolaev and V.A. Saleev, Phys. Rev. D 87, 014022 (2013).

[9] H.Jung et al., Eur. Phys. J. C 70, 1237 (2010).

[10] M. Ciafaloni, Nucl. Phys. B 296, 49 (1988);

S. Catani, F. Fiorani and G. Marchesini, Phys. Lett. B 234, 339 (1990); Nucl. Phys. B 336, 18 (1990); G. Marchesini, Nucl. Phys. B 445, 49 (1995).

[11] S.P. Baranov, A.V. Lipatov and N.P. Zotov, Phys. Rev. D 81, 094034 (2010).

[12] L.N. Lipatov and M.I. Wyazovsky, Nucl. Phys. B 597, 399 (2001); A.V. Bogdan and V.S. Fadin, Nucl. Phys. B 740, 36 (2006).

[13] M. Deak, H. Jung, K. Kutak, arXiv:0807.2403 [hep-ph].

[14] F. Hautmann, M. Hentschinski and H. Jung, Nucl. Phys. B 865, 54 (2012).

[15] S. Catani and F. Hautmann, Nucl. Phys.B 427, 475 (1994); Phys. Lett. B 315, 157 (1993).

[16] M. Ciafaloni and D. Colferai, JHEP 0509, 069 (2005).

[17] T. Affolder et al. (CDF Collaboration), Phys. Rev. Lett. 87, 131802 (2001).

[18] Abbott et al. (Dø Collaboration), Phys. Rev. Lett. 82, 4769 (1999). 
[19] G. Watt, A.D. Martin and M.G. Ryskin, Phys. Rev. D 70, 014012 (2004); ibid. D 70, 079902 (2004).

[20] A. Kulesza and W.J. Stirling, Nucl. Phys. B 555, 279 (1999).

[21] G.P. Lepage, J. Comput. Phys. 27, 192 (1978).

[22] T. Affolder et al. (CDF Collaboration), Phys. Rev. Lett. 84, 845 (2000). 